Ann. Biol. anim. Bioch. Biophys., I966, 6 (1), 93-100.

\title{
ÉQUILIBRE ACIDO-BASIQUE DU SANG ET FORMATION DE LA COQUILLE DE L'CEUF
}

\author{
P. MONGIN et L. LACASSAGNE \\ Station de Recherches avicoles, \\ Centre national de Recherches zootechniques, 78 - Jouy-en-Josas
}

SOMMAIRE

Nous avons mesuré sur le sang veineux de 8 poules domestiques le $\mathrm{pH}$, la $p \mathrm{CO}_{2}$ et la teneur en bicarbonates du sang au cours de la formation de la cocuille du deuxième ouf de la série. Cette formation a une incidence directe sur l'équilibre acido-basique du sang. Dès que l'œuf pénètre dans l'utérus une acidose se développe et atteint son maximum $22 \mathrm{~h}$ après l'oviposition précédente. A ce moment, on observe une chute de la bicarbémie partiellement compensée par une baisse de la $p \mathrm{CO}_{2}$. Ces variations doivent être attribuées au passage à travers l'utérus des bicarbonates nécessaires à la formation de la coquille comme le montre une étude parallèle effectuée sur 3 coqs pris comme animaux témoins.

Chez la poule domestique, la coquille de l'œuf renferme environ $5 \mathrm{~g}$ de carbonate de calcium dont $2 \mathrm{~g}$ de $\mathrm{Ca}^{++}$et $3 \mathrm{~g}$ de $\mathrm{CO}_{3}^{--}$. L'os, constitué essentiellement de phosphate représente une réserve de calcium, mais il ne peut fournir les ions carbonates nécessaires à la formation de la coquille.

GuTowska et MrTcheiL, en I945, à la suite d'une étude sur l'anhydrase carbonique, ont émis l'idée que les carbonates de la coquille proviennent des bicarbonates du sang. A notre connaissance, cette hypothèse n'a jamais été vérifiée. Si elle est fondée, l'équilibre acido-basique du sang devrait être perturbé durant la formation de la coquille puisque les bicarbonates constituent la majeure partie des réserves alcalines.

Nous avons donc entrepris l'étude des variations de la concentration en ions $\mathrm{CO}_{3} \mathrm{H}^{-}$, du $\mathrm{pH}$ et de la $p \mathrm{CO}_{2}$ dans le sang de la poule au cours de la sécrétion de la coquille $\left({ }^{1}\right)$.

(1) Le présent article complète la note déjà publiée (MONGIN et LACASSAGNE, I964). 


\section{MÉTHODE EXPÉRIMENTAIE}

\section{Animatux}

I.es observations ont été faites sur 8 poules et 3 coqs Rhode $\times$ Wyandotte $M .41$ âgés d'un an, laissés à demeure dans une pièce climatisée et habitués à la présence de l'homme. Les poules étaient placées dans des cages individuelles permettant l'enregistrement automatique de l'heure de ponte. Le régime lumineux était un nycthémère de $24 \mathrm{~h}$ comprenant is $\mathrm{h}$ de lumière et $10 \mathrm{~h}$ d'obscurité. Enfin les animaux étaient nourris ad libitum avec un ailment standard.

\section{Prilèuenents}

Les prélèvements de sang ont été faits sur la veine alaire gauche par la méthode de la canule permanente. A la suite d'une courte anesthésie locale à la xylocaïne à 2 p. Ioo, un cathéter de polyéthylène de $0,90 \mathrm{~mm}$ de diamètre fut mis en place de la manière suivante : dégarnie, la veine est percée à l'aide d'une fine aiguille hypodermique et le cathéter, effilé à son extrémité, est forcé dans cet orifice et introduit de $2 \mathrm{~cm}$ environ. Ensuite le cathéter est fixé dans le muscle adjacent par 2 fils et la peau est recousue. Il n'y a jamais de ligature, même temporaire, de la veine si bien que cette méthode n'entraine jamais l'arrêt de la circulation veineuse durant l'expérience.

La canule étant dirigée vers le couur, on obtient un écoulement naturel du sang par une légère pression sur la partie céphalique de la veine. Après charque prélèvement, on injecte $2 \mathrm{ml}$ d'une solution d'héparine à 1 p. I ooo dans la canule qui est ensuite fermée par un bouchon de cire. Le procédé évite la coagulation du sang car la canule est toujours remplie d'héparine.

Lors de la prise d'échantillon sur l'animal placé debout, on laisse la canule se vidanger de son héparine ( $1 \mathrm{ml}$ environ) puis on recueille le sang dans un capillaire de verre de façon telle qu'il n'y ait aucun contact avec l'air. Le capillaire, bouché aux extrémités par de la cire, renferme quelques cristaux d'héparine ainsi qu'une tige métallique de quelques millimètres qui permet, à l'aide d'un aimant, une dissolution homogène de l'héparine. Une telle préparation est toujours satisfaisante car elle évite la coagulation du sang ; la quantité préle vée est très faible : $80 \mu$ l. Les erreurs qui pourraient provenir d'une hémodilution sont ainsi évitées. Chaque prélèvement est constitué de 4 capillaires de verre numérotés de I à 4 , ce qui représente $0,320 \mathrm{ml}$ de sang.

Les prélèvements sont faits à heure régulière (fig. I) l'oviposition étant considérée comme temps zéro. Outre les stades essentiels de la formation des différentes parties de l'œuf, nous avons retenu le temps de $\mathbf{2 2} \mathrm{h}$ après l'oviposition car, au cours d'essais préliminaires, il nous est apparu que ce stade correspondait à la valeur minimum du pH.

\section{Méthodes de mesures}

Sur chaque prélèvement nous avons mesuré le pII, la $\mathrm{pCO}_{2}$ en millimètres de mercure et la concentration des ions bicarbonates en milliéquivalents par litre.

Le pH est mesuré à l'aide d'une microélectrode thermostatée à $4 \mathrm{I}, 5^{\circ} \mathrm{C}$ (Radiometer : type E $502 \mathrm{I}$ ) et d'un $\mathrm{pH}$-mètre (Radiometer : type 3) qui permet d'évaluer le 0,05 d'unité pH en valeur relative. Afin de tester l'homogénéité de chaque prélèvement, le pII réel est mesuré sur les capillaires $\mathrm{I}$ et 4 et lorsque ces deux valeurs diffèrent de plus de un centième d'unité, l'échantillon est rejeté. Dans cas le contraire, la valeur du capillaire 4 est considérée comme la valeur du pH réel.

La $p \mathrm{CO}_{2}$ est évaluée graphiquement par la méthode de SIGGAARD ANDERSEN (1962) en équilibrant le sang des capillaires 2 et 3 avec des $\mathrm{pCO}_{2}$ connues dans un tonomètre thermostaté (Radiometer : type AMT I). Pour ce faire, nous disposions de 2 bouteilles remplies d'un mélange oxygèneanhydride carbonique dans les proportions suivantes :

et

$$
\begin{array}{ll}
\mathrm{O}_{2}=96 \text { p. } 100 & \mathrm{CO}_{2}=4 \pm 0, \mathrm{I} \text { p. I00. } \\
\mathrm{O}_{2}=92 \text { p. } 100 & \mathrm{CO}_{2}=8 \pm 0, \mathrm{I} \text { p. I00. }
\end{array}
$$

$\mathrm{Au}$ cours de toute l'expérience, nous avons utilisé les mêmes bouteilles afin de ne pas cumuler 
les erreurs de composition des mélanges. La $p \mathrm{CO}_{2}$ du gaz issu de ces bouteilles était calculée de la manière suivante :

$$
p \mathrm{CO}_{2}=\frac{(\mathrm{B}-\mathrm{T}) \mathrm{CO}_{2} \%}{\text { roo }} \mathrm{mm} \text { de } \mathrm{Hg} .
$$

$\mathrm{B}$ : pression barométrique lue à chaque prélèvement en $\mathrm{mm}$ de $\mathrm{Hg}$.

$\mathrm{T}$ : tension saturante de vapeur d'eau à $4 \mathrm{I}, 5^{\circ} \mathrm{C}$ soit $60 \mathrm{~mm}$ de $\mathrm{Hg}$.

$\mathrm{CO}_{2} \%$ : teneur en $\mathrm{CO}_{2}$ de la bouteille de gaz. derson :

La concentration des ions bicarbonates est caculée à partir de l'équation d'Hasselbach Hen-

$$
\mathrm{pH}=p \mathrm{~K}^{\prime}+\log \frac{\mathrm{CO}_{3} \mathrm{H}^{-}}{a \cdot p \mathrm{CO}_{2}}
$$

$a=$ constance de solubilité du $\mathrm{CO}_{2}$ dans l'eau à $4 \mathrm{r}, 5^{\circ} \mathrm{C}$ soit $0,027^{8}$;

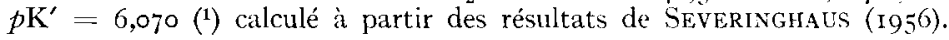

Dans le but de normaliser les résultats, nous nous sommes toujours astreints à faire nos observations durant la formation du $2^{\mathbf{e}}$ ceuf de la série.

De plus, il est à noter que les mesures sont toujours faites dans les $30 \mathrm{mn}$ qui suivent le prélèvement; on évite ainsi les méfaits de la conservation des échantillons sur les valeurs du pH (PORTNOY et coll., 1965). De même, pour la teneur utilisée, l'héparine n'avait aucun effet sur les mesures (SiggaARD ANDERSEN, 1960). Remarquons enfin que nous avons négligé le taux de saturation en oxygène de l'hémoglobine puisque les mesures ne comportaient pas de comparaison entre sang artériel et sang veineux.

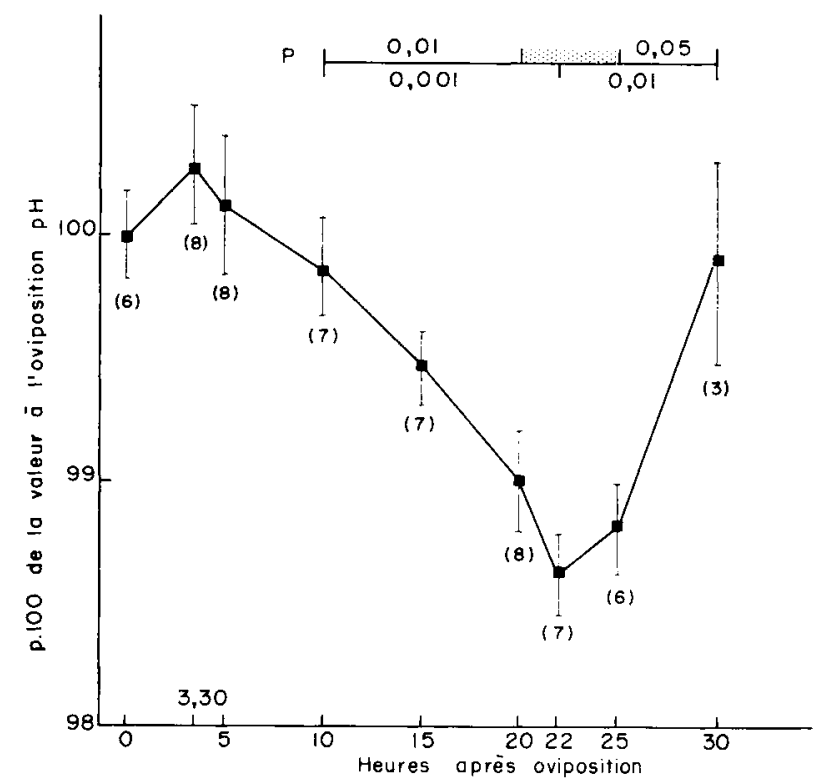

FIG. I. -- Variation du $p H$ veineux général durant la formation de l'ouf

Chaque point correspond à la moyenne $\pm \frac{\sigma}{\sqrt{n}}$ des observations dont le nombre est indiqué entre parenthèses. La valeur roo correspond à un $\mathrm{pH}$ de 7,52 .

$\mathrm{P}$ indique le seuil de signification de la différence entre 2 moyennes trouvées aux limites de l'intervalle de temps indiqué par 2 barres verticales. Les zones pointillées indiquent que la différence n'est pas significative au seuil de 5 p. 100 .

(1) Lorsque nous avons fait ce travail, nous n'avions pas encore connajssance des résultats de HeLBACKA et coll. (I964) qui ont mesuré le $p \mathrm{~K}^{\prime}$ sur le sang de la poule. Toutefois, notre estimation de $p \mathrm{~K}^{\prime}$ diffère très peu de la leur, compte tenu du fait que nous travaillons à $4 \mathrm{I}, 5^{\circ} \mathrm{C}$ au lieu de $4 \mathrm{I}^{\circ} \mathrm{C}$. 


\section{RÉSUI,TATS ET DISCUSSION}

L'évolution du $\mathrm{pH}$ veineux général (fig. I) fait apparaître une légère alcalose au moment où l'œuf passe dans le magnum et dans l'isthme. Ensuite, une acidose se développe régulièrement dès que l'œuf pénètre dans l'utérus. Elle atteint un maximum $22 \mathrm{~h}$ après l'oviposition, puis le $\mathrm{pH}$ revient à la valeur normale.

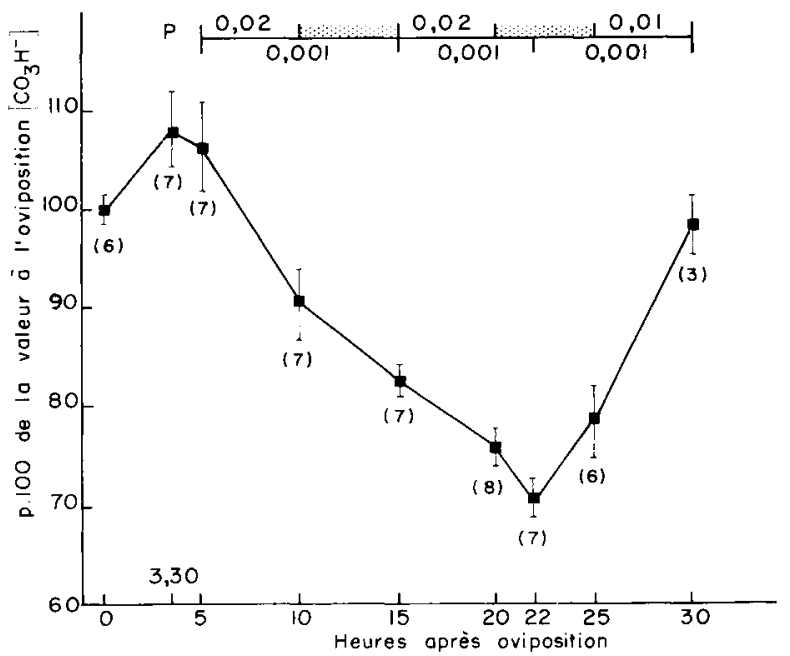

Fic. 2. - Variation de la concentration en bicarbonates du sang veineux général durant la formation de l'ouf

Voir légende de la figrure I.

La valeur 100 correspond à une bicarbémie de 29,6 méq/l.

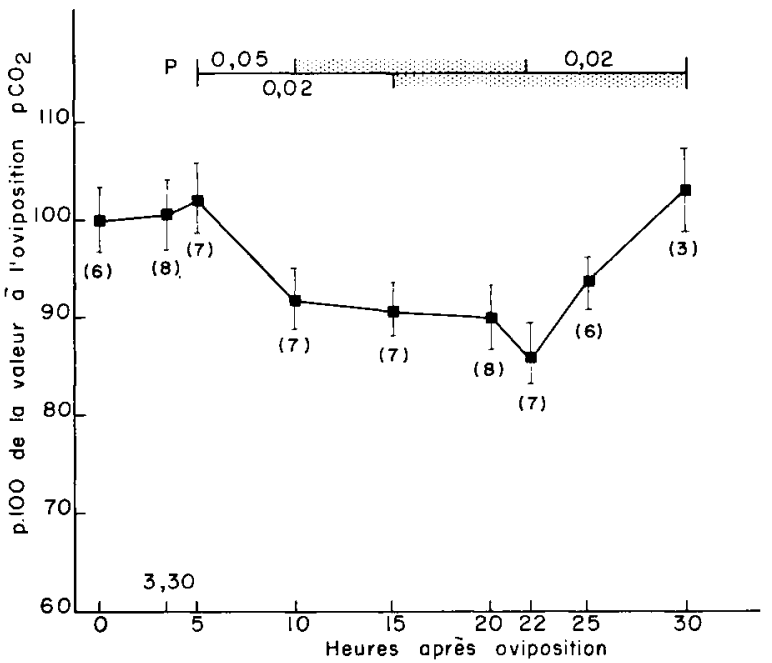

FIG. 3. - Variation de la pression partielle d'anhydride carbonique dans le sang veineux général durant la formation de l'ceuf Voir légende de la figure I.

$\mathrm{La}$ valeur 100 correspond à une $p \mathrm{CO}$ de $37,7 \mathrm{~mm}$ de mercure. 
La bicarbémie veineuse (fig. 2) suit la même évolution et chute de 30 p. Ioo $22 \mathrm{~h}$ après l'oviposition. De même, $1 \mathrm{a} p \mathrm{CO}_{2}$ (fig. 3) accuse une évolution parallèle, mais avec une amplitude moindre que celle des bicarbonates puisque la chute maximum est de 15 p. IoO.

Une représentation graphique (fig. 4) dans un système de coordonnées tridimensionnelles (DAVENPORT, I962) montre que l'évolution de l'équilibre acido-basique du sang entre 5 et $22 \mathrm{~h}$ après l'oviposition correspond à une acidose d'origine métabolique étant donné qu'il y a perte d'ions $\mathrm{CO}_{3} \mathrm{H}^{-}$. Cette acidose est partiellement compensée par une chute de la $p \mathrm{CO}_{2}$ sanguine.

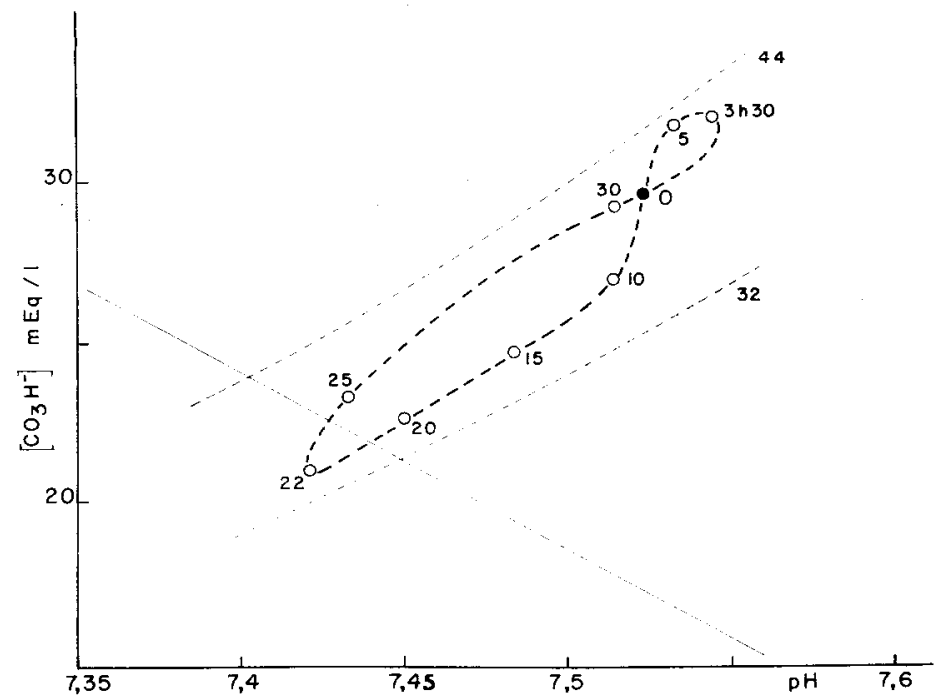

FIG. 4. - Variation de l'équilibre acido-basique du sang veineux durant la formation de l'cuf

-...-. ligne tampon normale du sang. donnée lorsque dans l'équation d'Hasselbach Henderson le $\mathrm{pH}$ et la concentration en bicarbonate varient). Les chiffres o indiquent le nombre d'heures après l'oviposition de l'œuf précédent.

Si l'on admet que cette perte d'ions bicarbonate se fait par voie rénale et ne représente rien d'autre qu'une variation diurne, on devrait retrouver ce phénomène chez le mâle.

Or, sur les 3 coqs Rhode $\times$ Wyandotte $M 41$ nous n'avons pas trouvé de variation de ce genre lorsque nous avons fait, d'une manière parallèle, la mesure de l'équilibre acido-basique du sang. A aucun moment de la journée, le pH, la $p \mathrm{CO}_{2}$ et la bicarbémie n'ont évolué de manière significative, si bien que pour déterminer la plage normale de variation de l'équilibre acido-basique chez le coq nous avons regroupé la totalité des 29 prélèvements effectués.

Il en est de même chez la poulette 7 jours avant la ponte du premier œuf (fig. 5).

Il apparaît alors que l'évolution de l'équilibre acido-basique du sang chez la poule en ponte est liée à la formation de l'œuf, et que la perte d'ions bicarbonate se fait au niveau de l'utérus puisque l'acidose se développe durant l'hydratation de 1'albumen et la formation de la coquille.

Lors de son hydratation dans l'utérus, l'albumen s'enrichit en ions et en particulier en bicarbonates qui seraient un des facteurs de sa qualité structurale. D'après

Annales de Biologie animale. - 1966. 
BRooks et PAGE (r938), il renferme en moyenne o,055 mole par litre soit environ Io7 $\mathrm{mg}$ de bicarbonates au total. On peut alors penser que le prélèvement de ces ions dans le sang est à l'origine de l'acidose qui commence à se développer $5 \mathrm{~h}$ après 1'oviposition. Il semble cependant plus vraisemblable d'admettre que c'est le $\mathrm{CO}_{2}$ qui diffuse vers l'albumen et non pas les bicarbonates car la $p \mathrm{CO}_{2}$, comme telle, agit sur la structure de l'albumen in vitro (BRooks et PAGE, I938) et in vivo (HELBACKA et coll., I964).

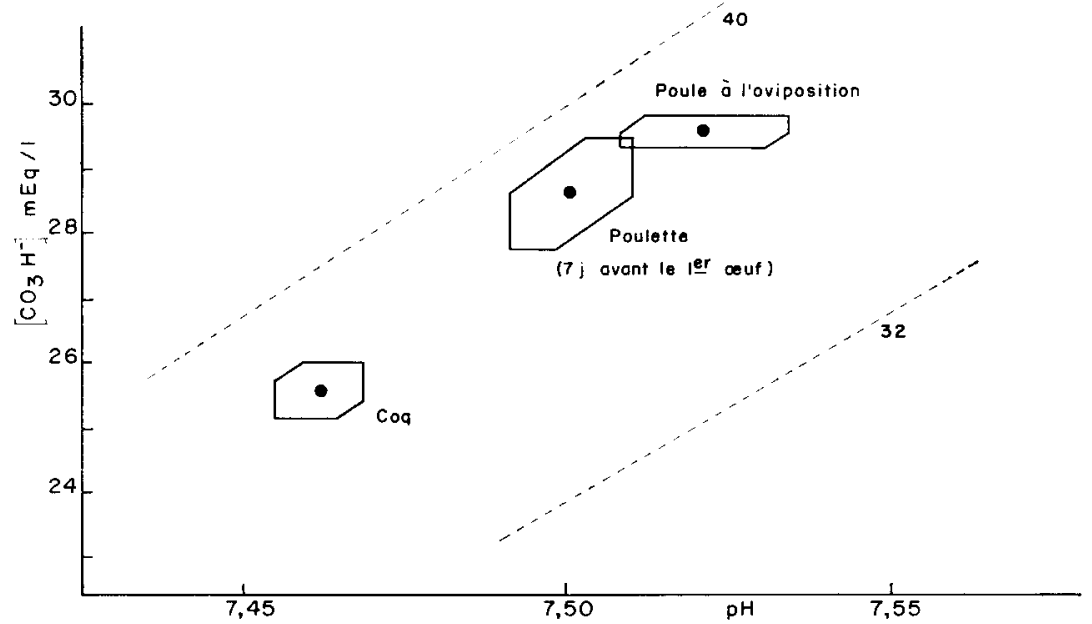

FIG. 5. - Plage normale de variation de l'équilibre acido-basique du sang, chez le coq, chez la pouletle 7 jours avant la ponte du $1^{e r}$ ouf, et chez la poule au moment de l'oviposition. ...... isobares de la $\mathrm{COO}_{2}$ (40 et $3^{2} \mathrm{~mm}$ de $\mathrm{Hg}$ )

A 1'opposé, il apparaît que pour la coquille ce sont les bicarbonates qui se trouvent à l'origine des carbonates selon le schéma de Gutowska et Mitchél, (I945).

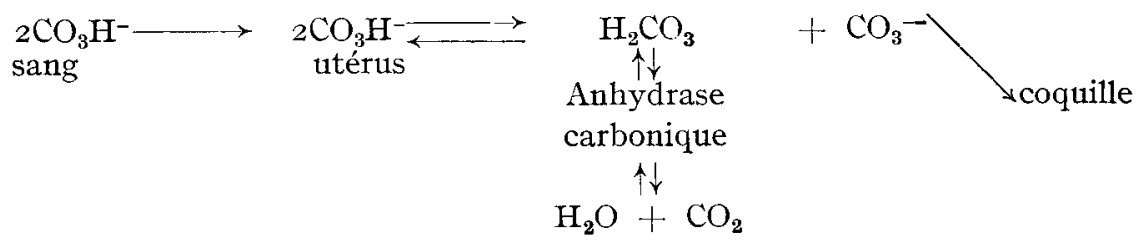

En étudiant l'anhydrase carbonique de l'utérus Diamanistein et Schluns (I964) ont en effet montré que l'ion $\mathrm{CO}_{3} \mathrm{H}^{-}$est sécrété dans la lumière de 1'utérus et cette présence de bicarbonates est chimiquement indispensable à la précipitation locale du carbonate de calcium sous forme de calcite (BACkra et coll., I963).

\section{CONCLUSIONS}

Io Si l'on compare les variations de la calcémie et de l'équilibre acido-basique du sang en liaison avec la formation de la coquille, on doit noter que la formation des carbonates est beaucoup plus éprouvante que la sécrétion du calcium. En effet, 
il est très difficile de mettre en évidence les variations de la calcémie dans la circulation générale, alors que la bicarbémie générale accuse en fin de calcification un déficit de $30 \mathrm{p}$. roo. On peut donc penser que tout facteur, capable de modifier l'équilibre acido-basique du sang, se répercute sur la formation de la coquille.

$2^{0} \mathrm{La}$ baisse de la $p \mathrm{CO}_{2}$ sanguine, indispensable au maintien $\mathrm{du} \mathrm{pH}$ dans les limites physiologiques, implique une régulation pulmonaire et une variation du débit ventilatoire en liaison avec le dépôt de la coquille. Cette conséquence normale, tirée des résultats précédents vient d'être vérifiée (MONGIN et LACASSAGNE, I965) et laisse présager une action possible du $\mathrm{CO}_{2}$ sur la formation de la coquille par voie pulmonaire.

$3^{\circ}$ Chez les vertébrés, la régulation de la bicarbémie se fait normalement par le rein qui réabsorbe plus ou moins des bicarbonates filtrés. Or chez la poule nous venons de montrer que l'utérus est aussi un organe d'excrétion des bicarbonates. I1 est donc vraisemblable qu'il s'établit un équilibre entre les deux, mais lors d'un stress le rein est-il prioritaire ou compétitif?

De plus, la formation de la coquille modifie l'équilibre normal des ions filtrés en prélevant en particulier, l'un des ions négatifs essentiels; l'équilibre se rétablit-il par les chlores ou les phosphates? Autant de questions relatives à la physiologie rénale que nous essayons actuellement d'éclaircir en liaison avec la formation de la coquille.

Reçu pour publication en décembre 1965 .

\section{SUMMARY}

BLOOD ACID-BASE BALANCE AND SHELL FORMATION

Venous blood $\mathrm{pH}, p \mathrm{CO}_{2}$ and bicarbonate concentration were estimated during the shell formation of the second egg in the clutch, on eight domestic hens. $p \mathrm{CO}_{2}$ was estimated according to SIgGAARD ANDERSEN's method and percentage of bicarbonates by means of HASSELBACH-HENDERSON's equation.

Shell formation has direct influence on blood acid-base balance. As soon as the egg enters the uterus, an acidosis arises and reaches a maximum 22 hours after the oviposition of the previous egg taken as time of reference. At this moment, we noticed a $3^{\circ}$ per cent bicarbonates drop, partly balanced by a $I_{5}$ per cent $p \mathrm{CO}_{2}$ decrease. These variations must be attributed to the excretion into uterus of the bicarbonates required by the shell formation, as shows a parallel investigation on 3 control cocks.

\section{RÉFÉRENCES BIBLIOGRAPHIQUES}

Bachra B. N., Trautz O. R., Simon S. L., I963. Precipitation of calcium carbonate and phosphate I. Spontaneous precipitation of calcium carbonate and phosphate under physiological conditions. Arch. Bioch. Biophys., 108, 124-1 38 .

Brooks J., PACE J. '938. The distribution of carbon dioxide in the hens's egg. Proc. roy. Soc. London (Série B), 126, I96-209.

Davenport H. W., I962. The A, B, C, of acid base chemistry. The University of Chicago Press.

Diamantstein T., Schluns J., rg64. Lokalisation und Bedeutung der Karboanhydrase in uterus von Legehennen. Acta Histochim, 19, 296-302. 
Gurowska M. S., Mitchell C. A., 1945 Carbonic anhydrase in the calcification of the egg shell. Poultry Sci., 24, I59-168.

Helbacka N. V., Casterline J. L., Smith C. J., 1963 . The effect of hight $\mathrm{CO}_{2}$ atmosphere on the laying hen. Poultry Sci., 42, 1082-1084.

Helbacka N. V., Casterline J. L., Smith C. J, Shaffner C. S., ig64. Investigation of plasma carbonic acid $p \mathrm{~K}^{\prime}$ of the chicken. Poultry Sci., 43, 138 -1 44 .

Mongin P., Lacassagne L., I964. Physiologie de la formation de la coquille de l'œuf de poule et équilibre acido-basique du sang. C R. Acad. Sci., Paris, 258, 3093-3094.

Mongin P., Lacassagne L., 1965. Physiologie de la formation de la coquille de l'œuf de poule et ventilation pulmonaire. C. R. Acad. Sci., Paris, 261, 4228-4229.

Mongin P., Lacassagne L., 1966 . Rythme respiratoire et physiologie de la formation de la coquille de l'œuf. Ann. Biol. anim. Bioch. Biophys., 6 (I), IoI-IIr.

Portnoy H. D., Thomas L. M., GurdjIan E. S., 1965. Storage of blood for measurement of $\mathrm{pH}, \mathrm{PO}_{2}$, $\mathrm{pCO}_{2}$. Clin. Chim. Acta, 11, 268-269.

Severinghaus J. W., Staffel M., Bradley A. F., i956. Variations of serum carbonic acid $p \mathrm{~K}^{\prime}$ with $\mathrm{pH}$ and temperature. J. appl. Phys., 9, 197-200.

SiggaArd Andersen O., Engel K., ig60. A new acid-base nomogram. An improved method for the calculation of the relevand blood acid-base data. Scand. J. Clin. Lab. Investig., 12, 177-196.

SiggaARd ANDERSEN O., I $96 \mathrm{r}$. Samplig and storing of blood for determination of acid-base status. Scand. J. Clin. Lab. Investig., 13, 196-204. 